\title{
Scaling of micro- and nanodevices actuated by Casimir forces
}

\author{
J. Bárcenas, L. Reyes, and R. Esquivel-Sirvent ${ }^{a)}$ \\ Instituto de Física, Universidad Nacional Autónoma de México, Ciudad Universitaria, D. F. 01000, México
}

(Received 27 September 2005; accepted 3 November 2005; published online 21 December 2005)

\begin{abstract}
The effect of the Casimir force in micro- and nanoelectromechanical systems is examined taking fully into account the dielectric properties of the materials, as well as the finite thickness of movable elements in micro- and nanosystems. The resulting equations are exact, and from the bifurcation diagrams the critical separation before jump-to-contact is determined. It is shown how the critical separation changes, for example, with the dielectric properties of the materials and how these systems can be rescaled based on the information from the bifurcation diagrams. (C) 2005 American Institute of Physics. [DOI: 10.1063/1.2152835]
\end{abstract}

One fundamental problem in micro/nanoelectromechanical systems (MEMS/NEMS) operation is the so-called "snap-down" effect, the permanent adhesion of moving parts when they are brought in close proximity to each other. These stiction phenomena can be of various origins, such as electrostatic interactions, capillary effects, or dispersive forces-such as van der Waals or Casimir forces. ${ }^{1}$ In all instances when a critical parameter is reached, a steady state or stable configuration of the device is not possible. Electrostatically actuated devices have been studied extensively and analytical expression for the stability conditions have been obtained, ${ }^{2,3}$ and also carried out for different systems, such as beams, membranes, and MEMS switches. ${ }^{4-6}$

The role of Casimir forces in the stability of MEMS and NEMS was first studied by Serry et ll $^{7}$ through a theoretical study of the deflection of a membrane strip. The material properties were expressed in an approximate way. ${ }^{7}$ Several authors have also studied the role of Casimir forces in the stability of micro- and nanosystems, using the expression originally derived by Casimir that assumed perfect conductors (infinite conductivity). ${ }^{8-11}$ Some references go as far as to (wrongly) state that the Casimir force is independent of material properties. Material properties, such as the electromagnetic response via the dielectric function, are important in the calculation of the Casimir force, as shown by Lifshitz. ${ }^{12}$

In this letter, we study the stability of NEMS and MEMS taking into account the full dependence of the material properties needed to calculate the Casimir force. Also, we show that by an appropriate selection of the material properties, the limitation on the size of MEMS and NEMS devices can be varied significantly.

The original derivation of the Casimir force considered two parallel plates made of a perfect conductor separated a distance $L$. The attractive force between the plates depended only on the separation and on fundamental constants as

$$
F_{0}=-\frac{A \hbar \pi^{2} c}{240 L^{4}}
$$

where $\hbar$ is Planck's constant, $A$ is the plate area, and $c$ is the speed of light in a vacuum. The parallel-plate configuration always yields an attractive Casimir force, ${ }^{13}$ although close cavities can experience repulsive forces depending on the

\footnotetext{
${ }^{a)}$ Electronic mail: raul@fisica.unam.mx
}

dimensions of the cavity. ${ }^{14}$ When dealing with real materials of finite conductivity, Lifschitz derived an expression for the force between two different half-spaces (labeled $j=1,2$ ) characterized by their dielectric function $\epsilon_{j}$. In modern notation, the Casimir force between two different slabs is given by ${ }^{15}$

$$
F=\frac{\hbar c}{2 \pi^{2}} \int_{0}^{\infty} Q d Q \int_{q>0} d k \frac{k^{2}}{q}\left(G^{s}+G^{p}\right),
$$

where $G_{s}=\left[r_{1 s}^{1} r_{2 s}^{-1} \exp (2 i k L)-1\right]^{-1}$ and $G_{p}$ $=\left[r_{1 s}^{1} r_{2 s}^{-1} \exp (2 i k L)-1\right]^{-1}$. In these expressions, the factors $r_{p, s}$ are the reflectivities for either $p$ - or $s$-polarized light, $Q$ is the wave vector component along the plates, $q=\omega / c$ and $k$ $=\sqrt{q^{2}+Q^{2}}$. When the reflectivities are replaced by the Fresnel coefficients, the original Lifshitz formula is recovered and Eq. (1) for ideal conductors is obtained when $\left|r_{p}\right|=\left|r_{s}\right|=1$ for both plates.

In this letter, we consider only MEMS and NEMS devices described by a one degree of freedom simple-lumped systems. Consider the system depicted in the inset of Fig. 1: Two parallel plates, one fixed and the other one attached to a spring of elastic constant $k$. Let $L_{0}$ be the equilibrium position of the moving plate. The equation of motion of the moving plate of mass $m$ is our coordinate system

$$
m \frac{d^{2} z}{d t^{\prime 2}}=-k(z)+F_{c}\left(L_{0}-z\right)
$$

where $F_{c}$ is the actuating force that is a function of the separation between the plates. By defining the dimensionless quantities $v=z / L_{0}$ and $t=\sqrt{\kappa / m} t^{\prime}$, we rewrite the last equation as

$$
\frac{d^{2} z}{d t^{2}}=-v+\frac{F_{c}(1-v)}{k L_{0}} .
$$

The condition for stability is obtained by setting the left-hand side of Eq. (4) equal to zero. Using the variable $v$, the initial (equilibrium) separation between the plates is for $v=0$, while contact between the plates corresponds to $v=1$. As an example that has been treated in the literature, let us first consider the Casimir force between ideal plates. By replacing Eq. (1) in the normalized equation of motion, Eq. (4), the stability condition is 


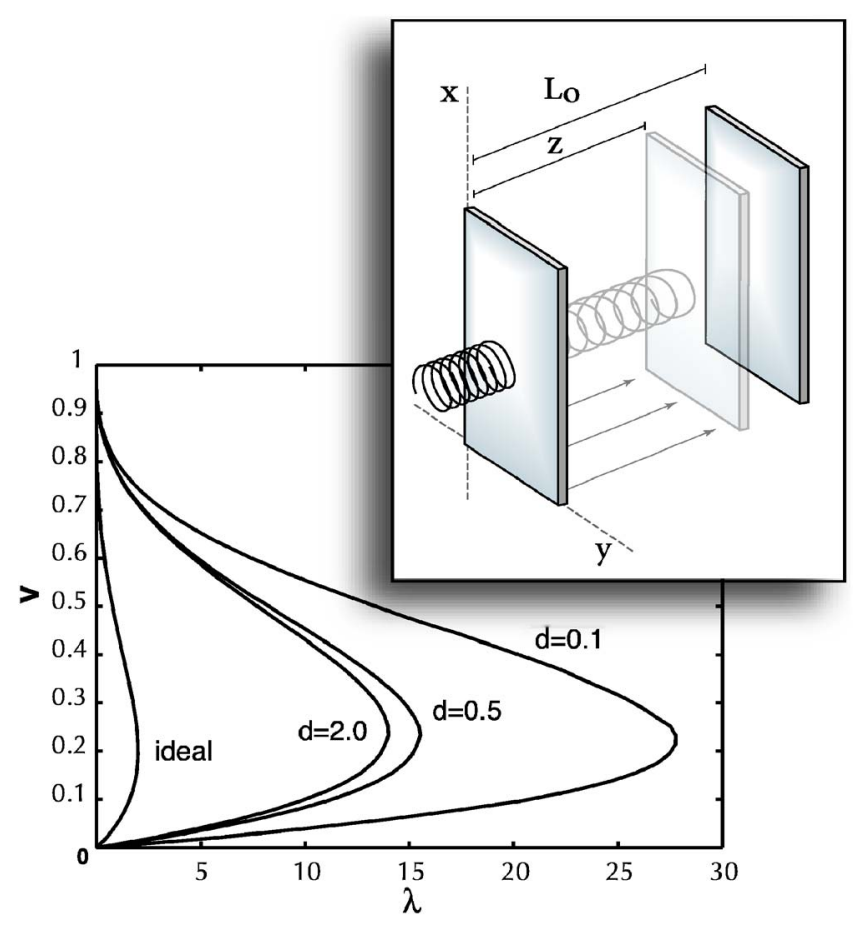

FIG. 1. Bifurcation diagram for Au films of different thicknesses. The thickness of the plate is normalized to $L_{0}$. The inset shows the configuration and coordinate system used.

$$
0=-v+\frac{\lambda}{(1-v)^{4}},
$$

where we define the bifurcation parameter $\lambda$ $=A \pi \hbar c /\left(240 L_{0}^{4}\right) k L_{0}$. Physically, this parameter gives the ratio of the Casimir force to the elastic force at the equilibrium position $L_{0}$. To fully introduce the material properties, we now assume that the plates in Fig. 1 are characterized by a frequency dependent dielectric function. Assume that one of the plates has a dielectric function $\epsilon_{1}(\omega)$, and the second plate $\epsilon_{2}(\omega)$. In general, the dielectric function can be nonlocal and also dependent on the wave vector. Here, we restrict the calculations to local dielectric functions, see for example, Refs. 16 and 17. Substitution of the force given by Eq. (2) into Eq. (4) gives

$$
0=-v+\lambda \frac{120}{\pi^{4}} \int_{0}^{\infty} \tilde{Q} d \tilde{Q} \int_{q>0} d \tilde{k} \frac{\widetilde{k}^{2}}{\widetilde{q}}\left(G(v)^{s}+G(v)^{p}\right) .
$$

The integrand has been written using the dimensionless variables and the wave vector components with a tilde are multiplied by $L_{0}$, for example $\tilde{k}=L_{0} k$.

Typical experimental measurements of Casimir forces involve Au-coated surfaces. We begin our analysis with two Au plates, both of thickness $d$. The thickness of the plate is normalized to $L_{0}$. Notice that the role of the thickness and the dielectric function obviously enters in the reflection coefficients. The Au slabs are modeled by a Drude-type dielectric function. The plasma frequency and damping were taken from Ref. 18. In Fig. 1, we present the bifurcation diagram for this case. That is, we solve for $\lambda$ as an implicit function of $v$ from Eq. (6) for different thicknesses of the slabs. For comparison, we plotted the resulting bifurcation when the plates are perfect conductors [Eq. (5)]. Figure 1 shows the characteristic behavior of the stability curves of singleDownloaded 04 Jun 2008 to 132.248 .209 .114 . Redistribution subje

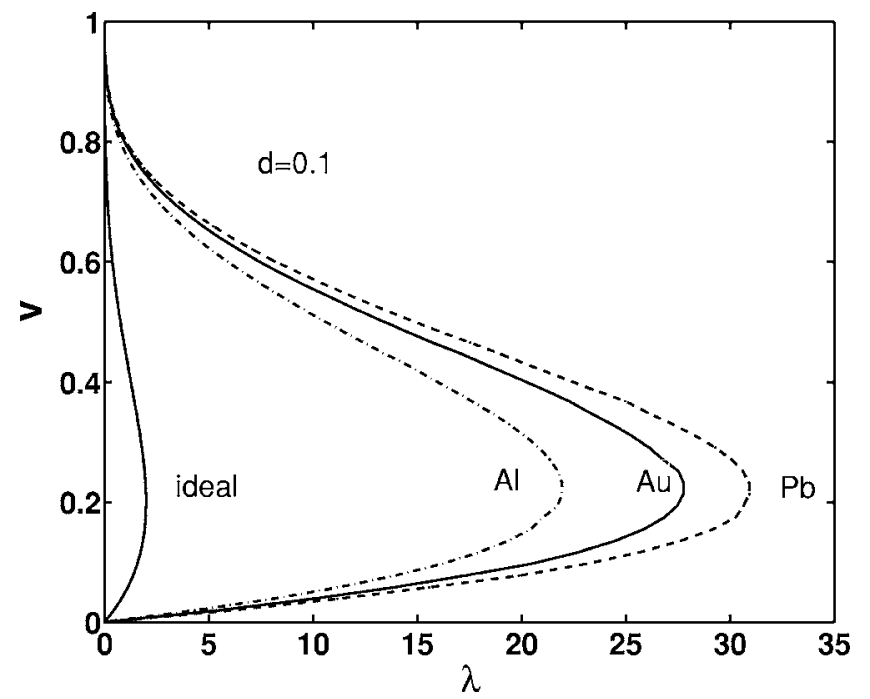

FIG. 2. Bifurcation diagram keeping the film thicknesses constant for different metals modeled using a Drude-type dielectric function.

lumped systems. The lower branch before the fold corresponds to the stable solutions, while the upper branch is unstable, thus the sudden jump to contact. The stability analysis can be found in Ref. 5 .

In all cases, the value of the dimensionless separation before snap down is $v=0.22$ and the critical value of the parameter $\left(\lambda_{c}\right)$ at which this occurs changes with the thickness. Similarly, we can keep the thickness of the slabs constant and change the dielectric function. As seen in Fig. 2, the shape of the bifurcation diagram is the same and, again, the critical separation before snap down is $v=0.22$. This is consistent with the fact that the critical separation depends only on the functional dependence of the actuating force with the plate separation; what changes is the corresponding critical value of $\lambda$.

The variation of the critical value of $\lambda$ with the thickness of the plates or the material properties allows for a rescaling of MEMS and NEMS devices. For example, let $\lambda_{0}$ be the maximum value before snap down for the ideal case, and let $\lambda_{i}$ be the critical value at snap down when the plates are made of thickness $d$. From the definition of $\lambda$, we can fix the value of the elastic constant, and we see that the equilibrium position when the $\mathrm{Au}$ plates have thickness $d$ is $L_{0 d}$ $=\left(\lambda_{i} / \lambda_{d}\right)^{1 / 5} L_{0 i}$, effectively reducing the equilibrium position.

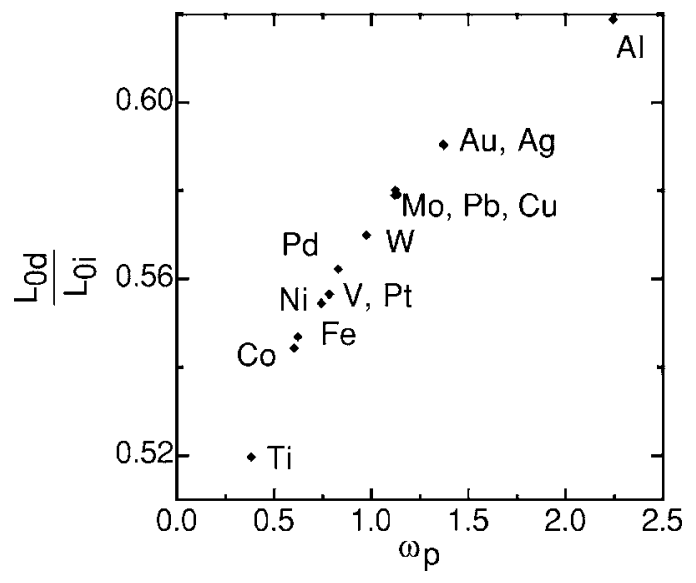

FIG. 3. Variation of the equilibrium position for a plate of finite thickness as

a function of the plasma frequency of different metals.
to AIP license or copyright; see http://apl.aip.org/apl/copyright.jsp 
In Fig. 3, we plotted the ratio $L / L_{0 i}$ for different metals. Hence, by using different materials, the equilibrium position is decreased significantly, therefore, allowing a reduction in the separation between movable parts in MEMS and NEMS.

The effect of a substrate can also be explored using Eq. (2) also. For example, if we assume that the fixed plate is deposited on a Si substrate and taking a value of $d=0.1$ for the plates, the critical value of $\lambda$ changes from 27.8 (no substrate) to 19.8 (with substrate). The one degree of freedom lumped system we have considered in this work might be limited. However several devices can be described by such an approximation; for example, membrane strips and torsional balances. ${ }^{9,19,20}$

In conclusion, we calculated the stability curves for a simple-lumped system actuated by a Casimir force taking into account the full dependence of this force on the dielectric properties. The advantage of using Eq. (2) as compared to approximate methods is that system parameters that determine the stability factors, such as the equilibrium position or spring constant, can be changed by selecting the dielectric properties of the materials. For example, the dielectric function of this system can be engineered using multilayered substrates.

Partial support from CONACyT-Mexico Grant No. 44306 and DGAPA-UNAM No. IN-101605.
${ }^{1}$ Y. P. Zhao, L. S. Wang, and T. X. Yu, J. Adhes. Sci. Technol. 17, 519 (2003).

${ }^{2}$ J. A. Pelesko, SIAM J. Appl. Math. 62, 888 (2002).

${ }^{3}$ G. Flores, G. A. Mercado, and J. A. Pelesko, Proceedings of IDETC/CIE, 19th ASME Biennial Conference on Mechanical Vibrations and Noise (ASME, New York, 2003), pp. 1-8.

${ }^{4}$ A. A. Chumak, P. W. Milonni, and G. P. Berman, Phys. Rev. B 70, 085407 (2004).

${ }^{5}$ J. A. Pelesko and D. H. Bernstein, Modeling MEMS and NEMS (Chapman and Hall/CRC, Boca Raton, FL, 2003).

${ }^{6}$ D. Jianning, M. Yonggang, and W. Shizhu, Sci. China (Series A) supplement 44, 255 (2001).

${ }^{7}$ F. M. Serry, D. Walliser, and G. J. Maclay, J. Appl. Phys. 84, 2501 (1998).

${ }^{8}$ J. G. Guo and Y. P. Zhao, J. Microelectromech. Syst. 13, 1 (2004).

${ }^{9}$ E. Buks and M. L. Roukes, Europhys. Lett. 54, 220 (2001).

${ }^{10}$ E. Buks and M. L. Roukes, Phys. Rev. B 63, 033402 (2001).

${ }^{11}$ G. Palazantzas, J. Appl. Phys. 97, 126104 (2005).

${ }^{12}$ E. M. Lifshitz, Sov. Phys. JETP 2, 73 (1956).

${ }^{13}$ It was recently speculated that high $\mu$ materials will yield a repulsive force in the case of parallel plates. O. Kenneth, I. Klich, A. Mann, and M. Ravzen, Phys. Rev. Lett. 89, 033001 (2002).

${ }^{14}$ S. Hacyan, R. Jauregui, and C. Villarreal, Phys. Rev. A 47, 4204 (1993).

${ }^{15}$ R. Esquivel-Sirvent, C. Villarreal, and W. L. Mochán, Phys. Rev. A 68, 052104 (2003).

${ }^{16}$ R. Esquivel, C. Villarreal, and W. L. Mochan, Phys. Rev. A 68, 052103 (2003).

${ }^{17}$ R. Esquivel-Sirvent and V. Svetovoy, Phys. Rev. A 69, 062102 (2004).

${ }^{18}$ M. A. Ordal, R. J. Bell, R. W. Alexander, L. L. Long, and M. R. Querry, Appl. Opt. 24, 4493 (1985).

${ }^{19}$ S. K. Lamoreaux, Rep. Prog. Phys. 68, 201 (2005).

${ }^{20}$ M. Bordag, U. Mohideen, and V. M. Mostepanenko, Phys. Rep. 353, 1 (2001). 